\title{
First head-to-head comparison of catheter and surgical ablation for drug-refractory AF
}

The FAST investigators have reported that surgical ablation (SA) is more effective than catheter ablation (CA) for the prevention of recurrent left atrial arrhythmias in patients with atrial fibrillation (AF) for whom medical therapy has been unsuccessful. However, the surgical approach was associated with an increased incidence of adverse events. "Our findings may lead to an upgrade of the SA position in the AF guidelines," comments Dr Lucas Boersma, who was lead author of the report published in Circulation, "although the risk of adverse events should not be neglected."

Minimally invasive SA for AF was first described in 2006 as an alternative to the open-heart Cox maze procedure. The FAST trial is the first randomized study in which this relatively new percutaneous surgical technique has been directly compared with the well-established intervention of radiofrequency CA.
The trial was conducted at two treatment centers; St Antonius Hospital in Nieuwegein, the Netherlands and the Hospital Clinic in Barcelona, Spain. Patients with paroxysmal or persistent AF refractory to at least one antiarrhythmic medication, and with dilatation of the left atrium, hypertension, or a failed prior catheter ablation, underwent $\mathrm{CA}$ $(n=63)$ or SA $(n=61)$. Surgery was performed under general anaesthetic using video-assisted thoracoscopy and involved pulmonary vein isolation, ablation of left atrial ganglia, and left atrial appendage excision. CA required only local anaesthesia and encompassed pulmonary vein isolation by wide-area linear antrum ablation.

At 6 months and 12 months follow-up, the rate of freedom from left atrial arrhythmias was higher in the SA group than in the CA group $(67.2 \%$ vs $44.4 \%$, $P=0.0178$ and $65.6 \%$ vs $36.5 \%, P=0.0022$, respectively). Surgery was most successful in patients with paroxysmal AF and in those who had previously undergone a failed CA procedure. This improved efficacy was, however, gained at the expense of a higher rate of adverse events associated with surgery $(23.0 \%$ vs $3.2 \%$ for procedural events, $P=0.001$ and $34.4 \%$ vs $15.9 \%$ at 12 months, $P=0.027$ ).

"Our work provides a scientific background to support the use of SA over CA in this specific population," concludes Dr Boersma; he suggests that this technique might be considered at an earlier stage in the management of AF. Indeed the investigators have already initiated the FAST-2 trial to compare SA with CA as first-line invasive therapy for paroxysmal AF.

\section{Alexandra King}

Original article Boersma, L. V. et al. Atrial fibrillation catheter ablation versus surgical ablation treatment (FAST): a 2-center randomized clinical trial. Circulation doi:10.1161/CIRCULATIONAHA.111.074047 